NASA Technical Memorandum 106290

AIAA-93-03252

, ....... CMOTT-93-11

\title{
Linear Instability of Curved Free Shear Layers
}

William W. Liou

Institute for Computational Mechanics in Propulsion and Center for Modeling of Turbulence and Transition Lewis Research Center Cleveland, Ohio

Prepared for the 3rd Shear Flow Control Conference sponsored by the American Institute of Aeronautics and Astronautics Orlando, Florida, July 6-9, 1993 


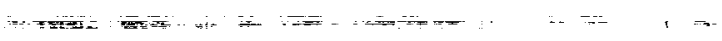

and 


\title{
LINEAR INSTABILITY OF CURVED FREE SHEAR LAYERS
}

\author{
William W. Liou \\ Institute for Computational Mechanics in Propulsion and \\ Center for Modeling of Turbulence and Transition \\ Lewis Research Center \\ Cleveland, Ohio 44135
}

\begin{abstract}
$\underline{\text { Abstract }}$
The linear inviscid hydrodynamic stability of slightly curved free mixing layers is studied in this paper. The disturbance equation is solved numerically using a shooting technique. Two mean velocity profiles that represent stably and unstably curved free mixing layers are considered. Results are shown for cases of five curvature Richardson numbers. The stability characteristics of the shear layer are found to vary significantly with the introduction of the curvature effects. The results also indicate that, in a manner similar to the Görtler vortices observed in a boundary layer along a concave wall, instability modes of spatially developing streamwise vortex pairs may appear in centrifugally unstable curved mixing layers.
\end{abstract}

\section{Introduction}

The hydrodynamic stability of slightly curved mixing layers is considered in this paper. Curved free shear layers exist in many problems with more generalized flow geometries, such as the backward facing step flow, flows with wall injection, and the flow inside side-dump combustors. It may be argued that a detailed examination of the stability characteristics and the structure of the curved free shear flow would provide a better understanding of the flow structures in the more complex problems.

The stability mechanisms of an incompressible curved shear layer may depend on the effects of the velocity difference and the streamline curvature. For a free shear layer with no streamline curvature, or a plane shear layer, the Kelvin-Helmholtz instability is the dominant instability mechanism ${ }^{1}$. In this case, two-dimensional disturbances are more unstable than three-dimensional disturbances ${ }^{2}$ and the Kelvin-Helmholtz instability introduces predominantly spanwise vortices. Rayleigh ${ }^{3}$ proved that the existence of an inflection point in the basic velocity profile was a necessary condition for the Kelvin-Helmholtz instability. For a curved free mixing layer with an inflectional velocity profile, it may be expected that the Kelvin-Helmholtz instability mechanism is still present.

In addition to the inflectional instability, the centrifugal instability can also influence the stability characteristics of a curved free mixing layer. The centrifugal force may either enhance or stabilize disturbances. The underlying mechanism is similar to the Taylor-Görtler instability. 
Taylor instability occurs in rotating flows, such as the Couette flow between two rotating coaxial cylinders. In this case, the instability produces steady toroidal vortices between the cylinders. Since the work of Taylor ${ }^{4}$, the stability of the flow between rotating cylinders has been widely studied. The existence of streamwise vortices in a boundary layer over a concave wall was first predicted by Görtler ${ }^{5}$ and re-examined later by Hall ${ }^{6}$. Görtler vortices appear in the form of counter rotating vortices in the streamwise direction. Rayleigh ${ }^{3}$ first showed that the necessary and sufficient condition for an inviscid flow to be centrifugally stable to axi-symmetric disturbance is that the square of the circulation should not decrease in the flow field. According to the Rayleigh circulation criterion, the centrifugal force may destabilize curved free mixing layers if the inner stream is faster. Therefore, both the Kelvin-Helmholtz and the centrifugal instabilities are relevant to the hydrodynamic stability of curved free mixing layers. Much attention has been focused on the individual instability mechanisms, as is evident by the great number of studies of these subjects. However, we are unaware of any previous reported work that has studied the hydrodynamic stability of free shear flows where both the Kelvin-Helmholtz instability and the centrifugal instability may be present and compete against each other. In the present analysis, the competing mechanisms of the Kelvin-Helmholtz and the centrifugal instabilities in curved free mixing layers is studied.

An instability analysis is also believed to be able to provide some insights into the turbulent structure of curved free mixing layers. This is especially true if the recent developments of the instability wave turbulence models are considered. These models describe the properties of the dominant, large-scale coherent structures in turbulent free shear flows as instability waves associated with the Kelvin-Helmholtz instability. It has been shown $n^{7,8,9}$ that the overall development of the coherent large-scale structures in plane and axisymmetric turbulent free shear flows may be well predicted by such instability wave models. The models are based on a weakly nonlinear theory with a description of the coherent large-scale structures as hydrodynamic instability waves. Using an integral analysis and assumed mean profiles, Morris et al. ${ }^{7}$ and Viswanathan and Morris ${ }^{8}$ successfully predicted the growth of plane and axisymmetric shear layers for a wide range of operating conditions. This includes the effects of velocity and density ratio as well as the free stream Mach numbers. Liou and Morris ${ }^{9}$ constructed a true turbulence closure scheme based on an instability wave model for the large-scale structures and successfully predicted the mean velocity of a plane mixing layer.

Large-scale spanwise vortical structures have been observed in both stably and unstably curved mixing layers ${ }^{10}$. These structures appear to be less two-dimensional in an unstably curved mixing layer than they are in a stably curved mixing layer. In addition, the Reynolds shear stress of an unstably curved free mixing layer has a higher peak level and a wider distribution in the low speed region than those in a plane mixing layer ${ }^{11}$. It is arguable that the centrifugal instabilities are responsible for the difference between the two cases. It should be noted that, according to 
the Rayleigh criterion, centrifugal forces stabilize the flow with a fast stream on the outside of the slow stream. Therefore, in this case, the Kelvin-Helmholtz instability should dominate. If the mixing layer is unstably curved, when the fast stream is on the inside of the slow stream, the centrifugal instabilities are likely to produce streamwise disturbances and, subsequently, change the characteristic structures in the flow. Therefore, a knowledge of the interaction mechanisms between the two different types of instability that exist in the curved free mixing layer should help to extend the application of instability wave models to such flows.

In the next section, the derivation of the governing equations for the disturbances is described. It is followed by a brief description of the basic velocity profile and the solution method used in the present analysis. Finally, some results of the calculations are presented and discussed.

\section{Formulation and Method of Solution}

\section{Stability Equations}

The flow geometry is shown in Figure 1. The curved free mixing layer is described in a cylindrical coordinate system, $(r, \theta, z)$. The instantaneous value of a flow variable, $\hat{\phi}$, is decomposed into a time-independent component denoted by the upper case and a fluctuation denoted by a prime. That is,

$$
\begin{aligned}
& \hat{v}^{*}\left(r^{*}, \theta^{*}, z^{*}, t^{*}\right)=v^{\prime *}\left(r^{*}, \theta^{*}, z^{*}, t^{*}\right) \\
& \hat{u}^{*}\left(r^{*}, \theta^{*}, z^{*}, t^{*}\right)=u^{\prime *}\left(r^{*}, \theta^{*}, z^{*}, t^{*}\right)+U^{*}\left(r^{*}\right) \\
& \hat{w}^{*}\left(r^{*}, \theta^{*}, z^{*}, t^{*}\right)=w^{\prime *}\left(r^{*}, \theta^{*}, z^{*}, t^{*}\right) \\
& \hat{p}^{*}\left(r^{*}, \theta^{*}, z^{*}, t^{*}\right)=p^{\prime *}\left(r^{*}, \theta^{*}, z^{*}, t^{*}\right)+P^{*}\left(r^{*}\right)
\end{aligned}
$$

where $v^{*}, u^{*}, w^{*}$ are the velocity components in the $\underline{r}^{*}, \theta^{*}$, and $z^{*}$ directions, respectively. $p^{*}$ denotes pressure.

For simplicity, the parallel flow assumption, commonly used in hydrodynamic stability analysis is applied. This approximation leads to the leading-order problem in a multiple scales method that includes the effects of the slow flow divergence ${ }^{12}$. Thus, in the current analysis, these effects are not considered.

Since the basic flow variation occurs mainly in the shear region, a streamline coordinate system is used. The transformation is,

$$
\begin{aligned}
r^{*} & =R^{*}+y^{*}, \\
r^{*} \mathrm{~d} \theta^{*} & =\mathrm{d} x^{*}
\end{aligned}
$$

where $R^{*}$ is the radius of curvature of a certain characteristic contour line of the curved mixing layer. In this analysis, it is taken as the contour line of the half velocity point of the basic velocity. 
For simplicity, the value of $R^{*}$ is assumed to be constant and much greater than the thickness, $\delta$, of the curved mixing layer. That is,

$$
\frac{\delta}{R^{*}}=\frac{1}{R} \ll 1
$$

The continuity and the momentum equations are nondimensionalized with respect to $\delta$ as the length scale and the velocity of the high speed stream as the velocity scale and linearized about the basic flow. Separable solutions are then sought for the fluctuations in the form,

$$
\left.\left\{\begin{array}{c}
u^{\prime} \\
v^{\prime} \\
w^{\prime} \\
p^{\prime}
\end{array}\right\}=\left\{\begin{array}{c}
u(y) \\
v(y) \\
w(y) \\
p(y)
\end{array}\right\} \exp [i(\alpha x+\beta z-\sigma t)]\right\}
$$

The $u, w, p$ variables may be eliminated from the linearized equations and a modified Rayleigh equation for the cross-stream velocity perturbation, $v(y)$, is found to be given by,

$$
\begin{gathered}
(\alpha U-\sigma) \frac{\mathrm{d}^{2} v}{\mathrm{~d} y^{2}}+\frac{2 \alpha U}{R} \frac{\mathrm{d} v}{\mathrm{~d} y} \\
-\left[(\alpha U-\sigma)\left(\alpha^{2}+\beta^{2}\right)+\alpha \frac{\mathrm{d}^{2} U}{\mathrm{~d} y^{2}}-\frac{2 U}{R} \frac{\beta^{2}}{\alpha U-\sigma} \frac{\mathrm{d} U}{\mathrm{~d} y}\right] v=0 .
\end{gathered}
$$

This equation is valid to the first order in $R^{-1} . \alpha$ and $\beta$ denote the wavenumbers in the streamwise and the spanwise directions and $\sigma$ is the frequency. The curvature terms in Eq.(5) are due to the centrifugal force alone. Equation (5) reduces to the Rayleigh equation ${ }^{3}$ for plane mixing flows as the curvature terms are neglected. If only streamwise vortices are considered, Eq. (5) reduces to the equation governing the inviscid stability of the flow along a curved wall ${ }^{13}$.

The disturbance kinetic energy equation can be written as

$$
\begin{gathered}
U \frac{\partial \overline{k^{\prime}}}{\partial x}=-\overline{u^{\prime} v^{\prime}}\left(\frac{\mathrm{d} U}{\mathrm{~d} y}-\frac{U}{R}\right)+\frac{\partial}{\partial x}\left(\overline{u^{\prime} k^{\prime}}\right)+\frac{\partial}{\partial y}\left(\overline{v^{\prime} k^{\prime}}\right) \\
+\frac{\partial}{\partial z}\left(\overline{w^{\prime} k^{\prime}}\right)+\frac{\overline{v^{\prime} k^{\prime}}}{R}-\frac{\partial}{\partial x}\left(\overline{u^{\prime} p^{\prime}}\right)-\frac{\partial}{\partial y}\left(\overline{v^{\prime} p^{\prime}}\right) \\
-\frac{\partial}{\partial z}\left(\overline{w^{\prime} p^{\prime}}\right)-\frac{\overline{v^{\prime} p^{\prime}}}{R}+\text { viscous terms }
\end{gathered}
$$

where $\overline{k^{\prime}}=\left(\overline{u^{\prime 2}+v^{\prime 2}+w^{\prime 2}}\right) / 2$. The curvature Richardson number, which is defined as (minus) the ratio of the kinetic energy production of the $v^{\prime}$-component to that of the $u^{\prime}$-component, can be written as,

$$
R_{f}=\frac{2 U}{R} /\left(\frac{\mathrm{d} U}{\mathrm{~d} y}+\frac{U}{R}\right)
$$

The Richardson number is a measure of the effects of the curvature of the mixing layer. The Richardson number is zero for a plane mixing layer. The physical boundary conditions used in this analysis are that the disturbances vanish in the far field. 


\section{Mean Velocity Profiles}

Two types of velocity distribution will be considered here. They are,

$$
\begin{array}{ll}
\text { Case } 1: & : \quad U(y)=\frac{1}{2}(1+\tanh (y)) \\
\text { Case 2 } & : \quad U(y)=\frac{1}{2}(1-\tanh (y))
\end{array}
$$

The profile based on the hyperbolic tangent function closely mimics the measured mean velocity profile of plane free mixing layers and has been widely used in the linear stability analysis of the plane free mixing layer ${ }^{1}$. For mildly curved mixing layers, the velocity profiles had been observed ${ }^{14}$ to have similar distributions to that of plane mixing layer. Consequently, Eq. (8) is likely to be a reasonable representation of the velocity profile of a curved free mixing layer. Case 1 represents the velocity profile of a stably curved mixing layer. That is, the high speed stream is on the outside of the low speed stream. Case 2 corresponds to a curved free mixing layer with the high speed stream on the inside of the low speed stream. In this case, in addition to the instability modes arising from the inflectional instability, the centrifugal instabilities may also be present.

The Richardson numbers, evaluated at the half velocity point, for Case $1(+)$ and Case 2(-) may be written,

$$
\left.R_{f}\right|_{U=0.5}=\frac{2}{R} /\left( \pm 1+\frac{1}{R}\right)
$$

From Eq. (5), the following forms of solution in the two cases can be obtained outside the shear layers in the regions of constant velocity,

Case 1:

$$
\begin{gathered}
v(y) \rightarrow \exp \left(\left[-\frac{\alpha}{R(\alpha-\sigma)} \pm\left(\frac{\alpha^{2}}{R^{2}(\alpha-\sigma)^{2}}+\alpha^{2}+\beta^{2}\right)^{\frac{1}{2}}\right] y\right) \\
\text { as } y \rightarrow \infty \\
v(y) \rightarrow \exp \left( \pm\left(\alpha^{2}+\beta^{2}\right)^{\frac{1}{2}} y\right) \\
\text { as } y \rightarrow-R
\end{gathered}
$$

Case 2:

$$
\begin{gathered}
v(y) \rightarrow \exp \left( \pm\left(\alpha^{2}+\beta^{2}\right)^{\frac{1}{2}} y\right) \\
\text { as } y \rightarrow \infty \\
v(y) \rightarrow \exp \left(\left[-\frac{\alpha}{R(\alpha-\sigma)} \pm\left(\frac{\alpha^{2}}{R^{2}(\alpha-\sigma)^{2}}+\alpha^{2}+\beta^{2}\right)^{\frac{1}{2}}\right] y\right) \\
\text { as } y \rightarrow-R
\end{gathered}
$$


The physical boundary conditions that the disturbances vanish far from the shear layer determine the proper signs to be used in the exponents. Equations (5) and (10) define an eigenvalue problem. In the spatial instability analysis described here, the complex streamwise wave number, $\alpha$, is the eigenvalue and the frequency, $\sigma$, is taken to be real. Solutions are sought that grow as the flow develops downstream.

\section{Numerical Scheme}

The modified Rayleigh equation (5) is integrated numerically using a fourth-order fixed stepsize Runge-Kutta scheme. A shooting procedure is used to solve for the eigenvalue, $\alpha$. On the concave side of the curved mixing layer, the integration starts away from the center of curvature at the edge of the shear layer. The stability code is an extension of a plane mixing layer stability $\operatorname{code}^{15}$, which has been validated against the results of Michalke and other numerical solutions. The initial guesses for the eigenvalues for the Kelvin-Helmholtz instability are taken as the eigenvalue for the corresponding plane mixing layer. For the centrifugal instability, the intial guesses for the eigenvalue are obtained by a grid search technique. The results of the spatial instability calculations are presented in the next section.

\section{Results and Discussions}

\section{Case1.}

Results for three different values of stable curvatures are shown for Case 1 . The values of $1 / R$ are $0.0,0.025$, and 0.05 . The corresponding Richardson numbers are $0,0.0488$, and 0.0952 , respectively. In this case, according to the Rayliegh circulation criteria, the centrifugal force generated by the streamline curvature works against the growth of disturbances and tends to stabilize the flow. The curved mixing layer, however, is still subject to the Kelvin-Helmholtz instability due to its inflectional velocity profile.

Figure $2 a$ shows the variation of the spatial growth rate with frequency of the two$\operatorname{dimensional}(2 \mathrm{D}) \mathrm{Kelvin}-\mathrm{Helmholtz} \operatorname{modes}(\beta=0)$. It can be seen that, as the curvature of the mixing layer increases, the spatial growth rate decreases. Similar trends can also be seen in Figure $2 \mathrm{~b}$, which shows the growth rate variation for oblique modes with $\beta=0.3$. For a plane mixing layer $\left(1 / R=R_{f}=0\right)$, it is known that the $2 \mathrm{D}$ modes are more unstable than the oblique modes of the same frequency. Oblique modes are also seen to be less amplified than $2 \mathrm{D}$ modes in a stably curved free mixing layer. As the curvature increases, the $2 \mathrm{D}$ modes become relatively more unstable than the oblique modes. For instance, for $1 / R=0.05$, the maximum growth rate of the oblique mode with $\beta=0.3$ is only approximately $60 \%$ of that of the $2 \mathrm{D}$ mode, compared to a relative value of $80 \%$ for the plane mixing layer where $1 / R=0$. 
Figure 2 also shows that, in the stably curved mixing layer, the Kelvin-Helmholtz modes are less unstable and the frequency range of the unstable modes is also reduced. Figure 3 shows the eigenfunction, $v$, of the oblique modes with $\beta=0.3$. The eigenfunctions are normalized such that $v(0)=1$. The distributions of the eigenfunctions change only slightly with curvature. Similar characteristics are also observed for the $2 \mathrm{D}$ modes in this case.

\section{Case2}

For this case, results are shown for two unstably curved free mixing layers. The values of the curvature are 0.025 and 0.05 . The corresponding $R_{f}$ are -0.0513 and -0.105 , respectively. The velocity profile used in this case simulates that of curved free mixing layers with the high speed stream on the inside of the low speed stream. As stated earlier, the centrifugal force is expected to further destabilize the mixing layer in this case. At each frequency, a hierarchy of unstable modes has been identified by the grid search study. Figure 4 shows the eigenvalue spectrum for $\beta=0.7$. For clarity, only the modes with the three highest growth rates are included. The phase velocity, $\sigma / \alpha_{r}$, of the mode with the highest growth rate, or mode 1 , maintains a value of approximately one half of the mean free stream velocity and varies only slightly with frequency. This is not the case for the other two modes, denoted by mode 2 and mode 3 . The growth rates of the mode 2 and mode 3 are much lower than those of the mode 1 . The modes 1,2 , and 3 with $\sigma=0.05$ are marked mode A, B, and C in Figure 4a, respectively. Their flow parameters can be found in Table 1.

Table 1. Mode parameters

\begin{tabular}{|c|c|c|c|c|}
\hline mode & $\beta$ & $\sigma$ & $\alpha_{r}$ & $-\alpha_{i}$ \\
\hline A & 0.7 & 0.05 & 0.09298 & 0.18087 \\
\hline B & 0.7 & 0.05 & 0.09310 & 0.05250 \\
\hline C & 0.7 & 0.05 & 0.07618 & 0.02328 \\
\hline D & 0.7 & 0 & 0 & 0.18885 \\
\hline E & 0.7 & 0 & 0 & 0.12470 \\
\hline F & 0.7 & 0 & 0 & 0.09478 \\
\hline G & 0.7 & 0 & 0 & 0.05220 \\
\hline
\end{tabular}

The vorticity patterns of the modes $\mathrm{A}, \mathrm{B}$, and $\mathrm{C}$ were studied. The results are presented in Figure 9. It will be shown that the mode 1 is actually the Kelvin-Helmholtz mode being influenced by the centrifugal forces. On the other hand, the mode 2 and the mode 3 show characteristics of both the Kelvin-Helmholtz instability mode and the centrifugal instability mode.

The effects of the streamline curvature on the mode 1 can be seen in Figure 5. Figure 5a shows the variation of the spatial growth rate of the $2 \mathrm{D}$ modes with frequency. The frequency range and the growth rates of the unstable modes are both seen to increase with increasing curvature. This is 
also true for the oblique modes. The growth rate predictions for the modes with $\beta=0.5$ are shown in Figure 5b. The unstable curvature has enhanced the Kelvin-Helmholtz instability uniformly over the entire unstable frequency range. Figure 6 shows the spatial growth rate for the KelvinHelmholtz modes with different spanwise wavenumbers. The curvature is set to $1 / R=0.05$. Figure 6 reveals some very interesting characteristics of the effects of curvature on the Kelvin-Helmholtz instability. The growth rates of the $2 \mathrm{D}$ modes are not uniformly higher than that of the oblique modes. For waves with large spanwise wave number, the growth rates of the $2 \mathrm{D}$ modes are lower than those of the oblique modes at low frequency. It should be recalled that in Case 1, where the high speed stream is on the outside of the low speed stream, the $2 \mathrm{D}$ modes remain dominant as the curvature changes.

Figure 6 shows that the effects of the unstable curvature on the Kelvin-Helmholtz modes are highly dependent on the frequency and the spanwise wavenumber. In the absence of curvature effects, the growth rates are uniformly zero for all the Kelvin-Helmholtz mode of zero frequency. With curvature, the spatial growth rates at zero frequency are finite and their absolute values increase with increasing wave angles. The growth rate of the spatially developing, time-independent $(\sigma=0)$ oblique mode equals that of the most unstable 2D mode at a certain wave angle, beyond which the time-independent oblique modes become the most unstable modes.

This observation leads to a study of the characteristics of the zero frequency or timeindependent unstable modes. Again, a grid search technique has been used to determine the possible locations of eigenvalues in the complex wave number plane. This has been done for different values of the spanwise wavenumber, $\beta$, and curvature, $1 / R$. The observed eigenvalues are then further refined by use of the shooting technique. The growth rate results are shown in Figure 7. It should be noted that, for modes with zero frequency, $\alpha_{r}=0$. That is, these modes are spatially amplifying; yet they assume no periodicity in time or in the streamwise direction. Consequently, from Eq.(10), solutions of the modified Rayleigh equation, that decay exponentially in the far field, exist only for $\beta^{2}>\alpha_{i}^{2}$. Indeed, no eigenvalues can be identified in the grid search process for $\beta^{2}<\alpha_{i}^{2}$. For $\sigma=0$, a series of eigenvalues can be identified in the grid search study for each value of the spanwise wavenumber, $\beta$. Figure 7 shows only the modes with the three highest growth rates. They appear to vary in a nearly linear fashion with the spanwise wavenumber. Instability modes with similar characteristics were also found in boundary layers along concave wall. Denier ${ }^{16}$ studied the inviscid, spatially growing Görtler modes in a boundary layer and found that their growth rates varied linearly with the spanwise wavenumber. Therefore, the behavior of these modes are likely to be associated with the centrifugal instability. It should be noted that the modes marked D, E, and F in Figure 7 are in fact the mode 1, the mode 2, and the mode 3 with $\sigma=0$, respectively. The flow parameters of the modes D-F are summarized in Table 1 .

Compared to a plane mixing layer, the unstable curvature is seen to not only enhance the 
Kelvin-Helmholtz modes but also generate new modes. Some questions one may ask immediately include: how do the Kelvin-Helmholtz modes change from a plane to an unstably curved mixing layer and how are the new modes different from the Kelvin-Helmholtz modes, particularly in their streamwise and spanwise structure? Since these new modes are generated by the centrifugal instability, do they represent streamwise structures similar to the Görtler vortices that may develop in unstably curved boundary layers? To attempt to answer these questions, the production of the wave kinetic energy and the vorticity patterns of these new modes have been examined. The distributions of the production term, which is the first term on the right hand side of Eq.(6), of the modes $\mathrm{D}, \mathrm{E}$, and $\mathrm{F}$ are shown in Figure 8. Note that the mixing layer is unstably curved and the eigenfunctions are normalized such that $v(0)=1$. For clarity, the wave energy production of the modes $\mathrm{D}$ and $\mathrm{E}$ have been multiplied by a factor of five. Figure 8 shows that there are one, two, and three peaks in the production profiles of the modes, D, E, and F, respectively. The production profile for a typical Kelvin-Helmholtz mode in a plane mixing layer, say for the most unstable mode, shows one positive peak and a small region of negative production on the low speed region. Therefore, these peaks in the disturbance energy production profiles of the curved mixing layer are indicative of possible coherent local events. Coherent local events may exist in the region where the production of disturbance kinetic energy is strong. This can be demonstrated by examination of the corresponding flow patterns.

Figure 9 shows the disturbance streamwise vorticity contours, $\omega_{x}$, over one wavelength for the $\sigma=0$ modes D, E, F and G. For comparison, the $\omega_{x}$ contour plots for the modes A, B, and C are also included. The spanwise wavenumbers of the mode $A-G$ are the same. It should be noted that there is a smooth transition between modes $A, B$ and $C$, and D, E and F, respectively. This implies that, for example, the mode $A$ and $D$ are the same mode at different frequencies. Contours of positive and negative levels are denoted by solid and dashed lines, respectively. The $\omega_{x}$ contours of the mode $\mathrm{A}$ are similar to those of the Kelvin-Helmholtz mode in a plane mixing layer, showing staggered vortices of opposite signs. These vortices extend across the region of substantial mean shear. Compared with the mode $A$, the vortices associated with the mode $B$ are elongated in the spanwise direction; such that regions of three vortices of opposite signs, partially stacked across the mixing layers, are formed. Likewise, the vorticity contours of the mode $\mathrm{C}$ are further elongated and show similar patterns to that of the mode $B$. While the vorticity contour patterns of all three modes, including the modes A, B, and C, are similar, the spatial growth rate of the mode $\mathrm{A}$ is four and eight times that of the modes $B$ and $C$, respectively.

Figures $9 \mathrm{~d}-9 \mathrm{f}$ show the $\omega_{x}$ contour plots of the modes D, E, and F. The $\omega_{x}$ contours of the mode $\mathrm{D}$ suggests that this mode is a pair of counter-rotating streamwise vortices, similar to the Görtler vortices observed in a boundary layer with a concave or unstably curved wall. Figure $9 \mathrm{e}$ shows that the disturbances of the mode $\mathrm{E}$ appears as two pairs of counter-rotating vortices 
stacking on top of each other with the neighboring vortices rotating in the opposite directions. Not surprisingly, the mode $F$, which has the third highest growth rate among the hierarchy of the zero-frequency $\beta=0.7$ modes is associated with disturbances with three counter-rotating vortex pairs with neighboring vortices rotating in the opposite directions. This is shown in Figure $9 f$. The trend of the increasing number of counter-rotating vortex pairs being associated with modes of increasingly lower growth rates is seen to continue. Figure $9 \mathrm{~g}$ show the $\omega_{x}$ contours of the mode G. Up to six counter-rotating vortex pairs can be identified. Based on what is known about the first three modes, it can be expected that a total of six peaks can be found in the profile of the wave kinetic energy production of this mode. It is also interesting to note that the locations of the vortex pair structures of the various modes shown in Figures $9 \mathrm{~d}-9 \mathrm{~g}$ are all biased toward the low speed side of the curved mixing layer. Like the spatially growing Görtler instability, the modes D-G are also spatially amplifying and have no periodicity in time.

Figure 10 shows the $\omega_{z}$ contour plots for only the modes A, B, and C. The streamwise wavenumbers of the mode $\mathrm{D}, \mathrm{E}$, and $\mathrm{F}$ are zero. For clarity, the plots are stretched in the $y$ direction. The contours show patterns similar to that of Kelvin-Helmholtz instabilities in a plane mixing layer, showing staggered vortices of opposite signs. For the modes $B$ and $C$, the spanwise vorticity is concentrated around the inflection point of the mean velocity profile.

\section{Summary}

The effects of streamline curvature on the stability of free mixing layers has been studied. With an inflectional mean velocity profile, a plane mixing layer is subject to the Kelvin-Helmholtz instability. When a mixing layer is stably curved, the curvature reduces the growth rate of the Kelvin-Helmholtz modes over the whole unstable frequency range. If the mixing layer is unstably curved, the Kelvin-Helmholtz modes become more unstable. In addition, various new modes are identified in the eigenvalue grid search study. These include a hierarchy of zero frequency modes which appear as layers of counter-rotating vortex pairs. Another group of the new modes bears some resemblance to both the Kelvin-Helmholtz mode and the centrifugal mode. The present study shows that, when the mixing layer is unstably curved, spatially growing, time-independent modes, similar to the Görtler vortices that are observed in a boundary layer along a concave wall, may also develop in the unstably curved free mixing layer.

\section{Acknowledgements}

The author would like to thank Professor Philip J. Morris at the Penn State University for his encouragement to pursue this study and his critical comments. 


\section{References}

1 Michalke, A., "On spatially growing disturbances in an inviscid shear layer," J. Fluid Mech. 23, 521 (1966).

2 Squire, H. B., "On the stability of three-dimensional disturbances of viscous flow between parallel walls," Proc. Roy. Soc. A, 142, 621-628 (1933).

3 Rayleigh, L., "On the stability, or instability of certain fluid motions," Proc. London Math. Soc. 11, 57-70 (1880).

4 Taylor, G. I., "Experiments with rotating fluids," Proc. Camb. Phil. Soc. 20, 326-329 (1921).

5 Görtler , H., "On the three-dimensional instability of laminar boundary layers on concave walls," NACA TM 1375 (1940).

6 Hall, P., "The Linear Development of Görtler Vortices in Growing Boundary Layer," J. Fluid Mech., 130, 41-57 (1983).

7 Morris, P. J., Giridharan, M. G., and Lilley, G. M. "On the turbulent mixing of compressible free shear layers," Proc. Roy. Soc. of London, Series A, 431, 219 (1990).

8 Viswanathan, K. and Morris, P. J. "Predictions of turbulent mixing in axisymmetric compressible shear layers," AIAA Journal, 30, 1529 (1992).

9 Liou, W. W. and Morris, P. J. "Weakly nonlinear models for turbulent mixing in a plane mixing layer," Phys. Fluids, 4, 2798 (1992).

10 Wang, C., "The effects of curvature on turbulent mixing layers," $\mathrm{Ph}$. D. Thesis, California Institute of Technology (1984).

11 Wyngaard, J. C., Tennekes, H., Lumley, J. L., and Margolis, D. P." Structure of turbulence in a curved mixing layer," Phys. Fluids, 11, 1251 (1968).

12 Morris, P. J., "Stability of a Two-Dimensional Jet," AIAA Journal, 19, 857-862 (1981).

13 Reid, W. H., "Inviscid modes of instability in flow over a concave wall," Journal of Mathematical Analysis and Applications, 2, 419 (1961).

14 Gibson, M. M. and Younis, B. A., "Turbulence measurements in a developing mixing layer with mild destabilizin curvature," Experiments in Fluids, 1, 23-30 (1983).

15 Liou, W. W. and Morris, P. J., "The eigenvalue spectrum of the Rayleigh equation for a plane shear layer," Int. J. Num. Fluids., 15, 1407 (1992).

16 Denier, J. P., Hall, P., and Seddougui, S. O., "On the receptivity problem for Görtler vortices: vortex motions induced by wall roughness," Phil. Trans. R. Soc. Lond. A. 335, 51-85 (1991). 


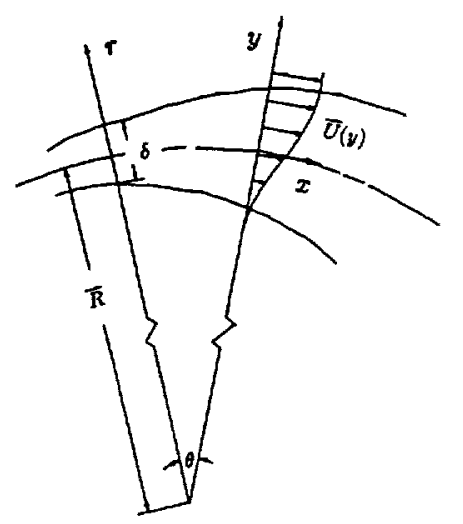

Figure 1. A sketch of curved free shear layer.

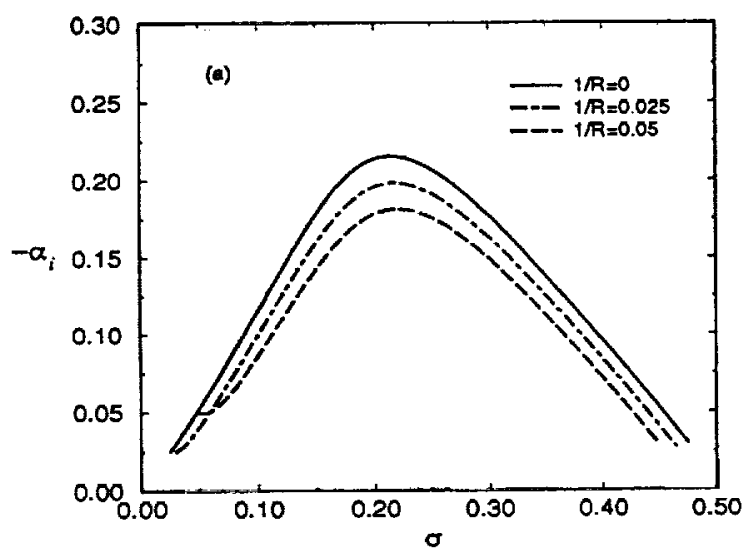

Figure 2. Variation of growth rate with frequency for the Kelvin-Helmholtz modes. Case 1.

(a) $\beta=0$; (b) $\beta=0.3$.

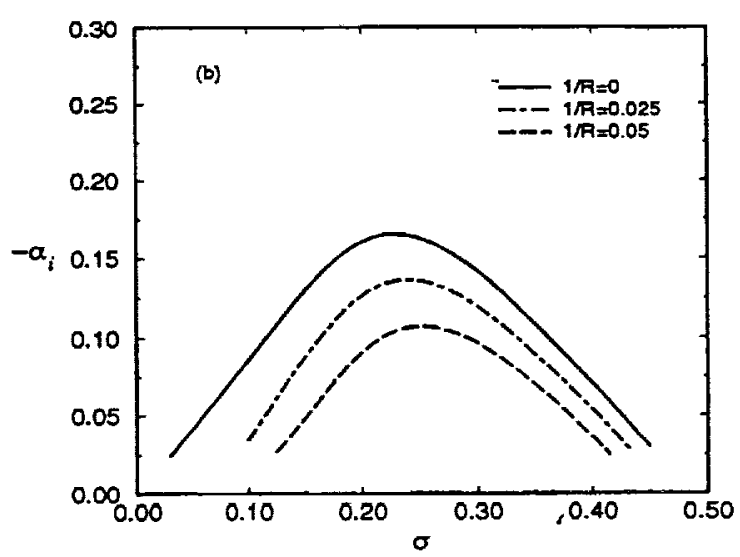

Figure 2. Continued

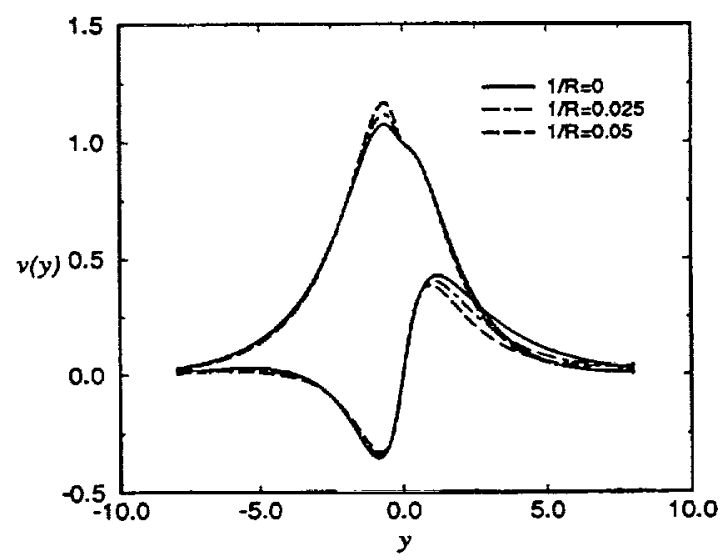

Figure 3. Distributions of the eigenfunction $v(y)$ for the most unstable Kelvin-Helmholtz modes with $\beta=0.3$. Case 1 . 

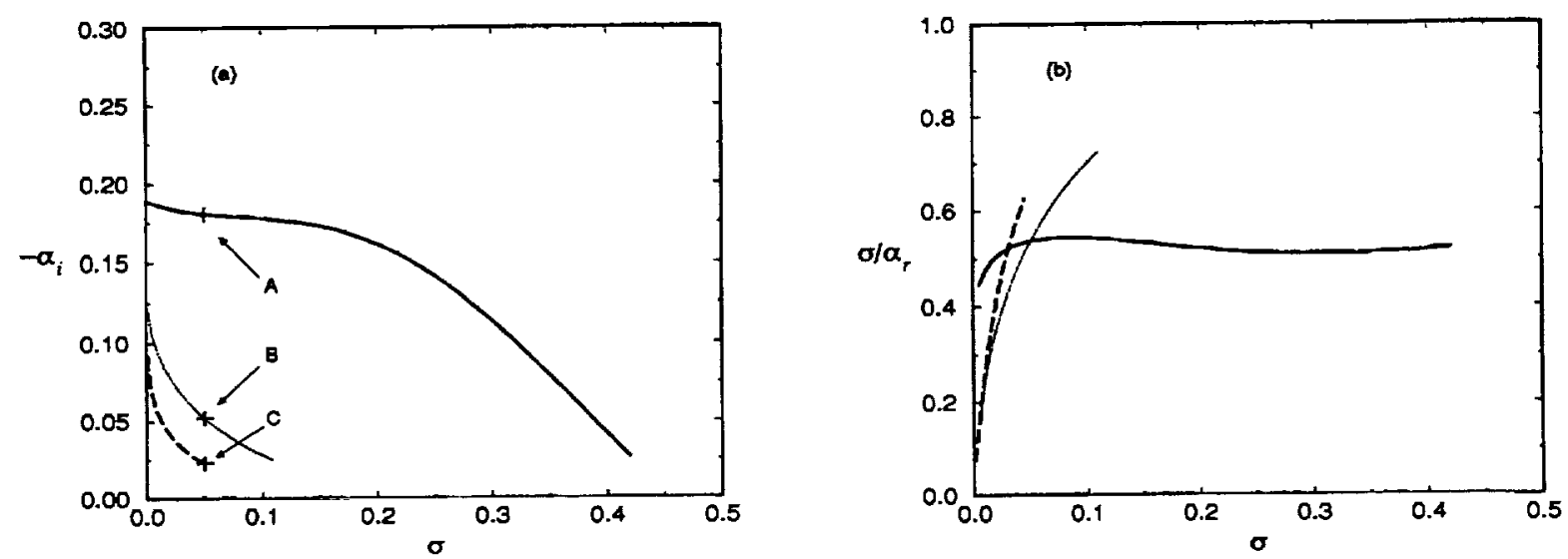

Figure 4. Eigenvalues for oblique modes with $\beta=0.7$. Case $2.1 / R=0.05$. (a) spatial growth rate; (b) phase velocity.,- mode $1 ; \ldots . .$. , mode $2 ;---$, mode 3 .
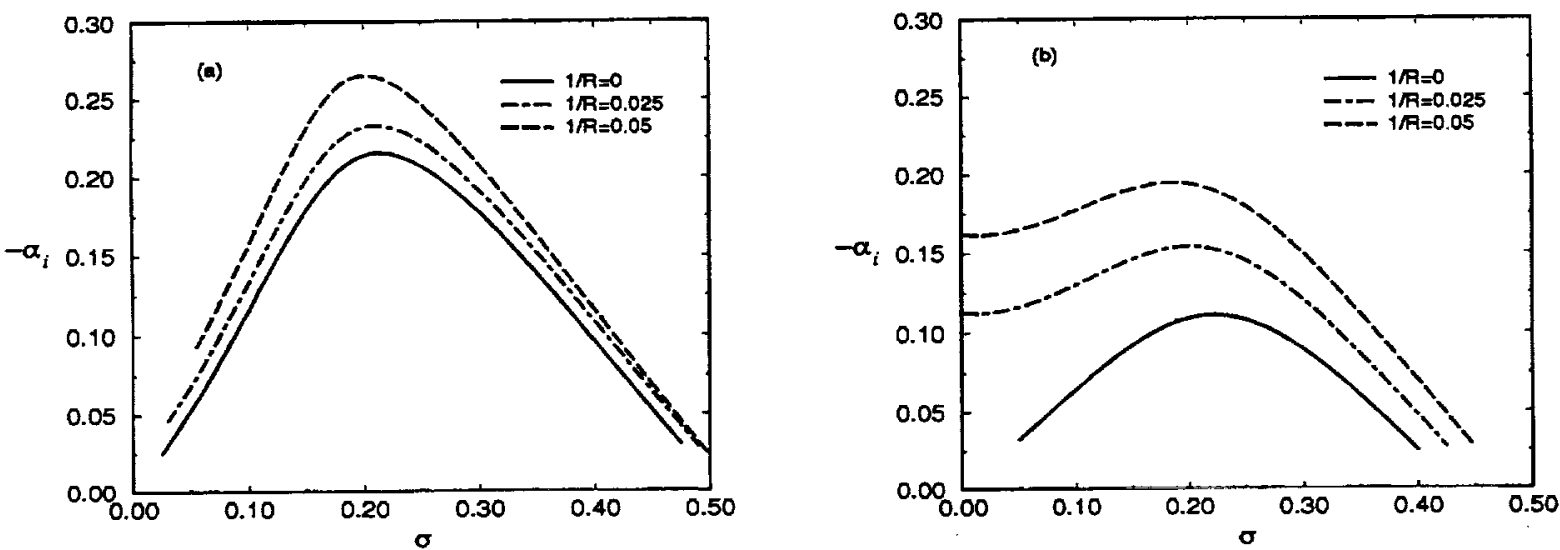

Figure 5. Variation of growth rate with frequency for the modes with the highest growth rates at each frequency for different curvature $1 / R$. Case 2 . (a) $\beta=0 ;(\mathrm{b}) \beta=0.5$.

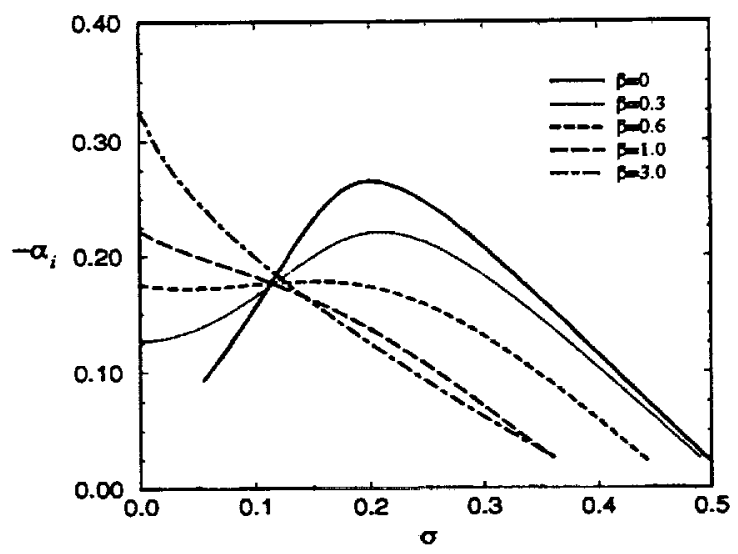

Figure 6. Variation of growth rate with frequency for the modes with the highest growth rates at each frequency for different spanwise wave number $\beta$. Case $2.1 / R=0.05$ 


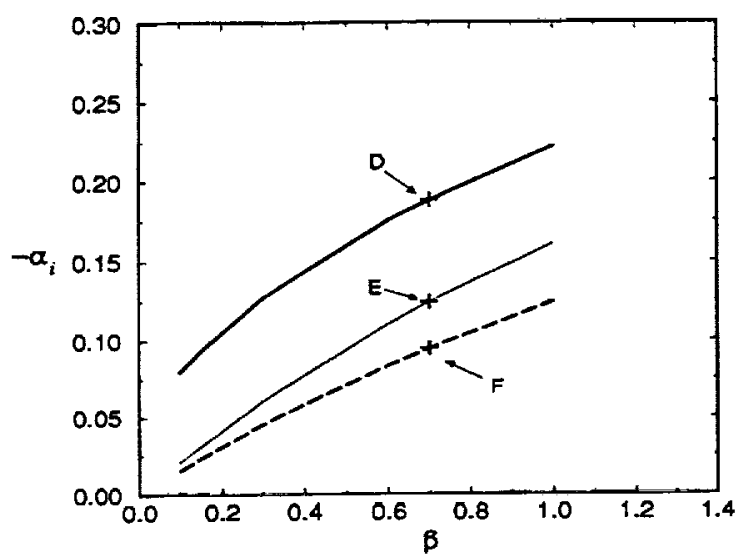

Figure 7. Variation of growth rate with spanwise wavenumber of zero-frequency modes. Case 2. $1 / R=0.05$. Legend: see Figure 4 .

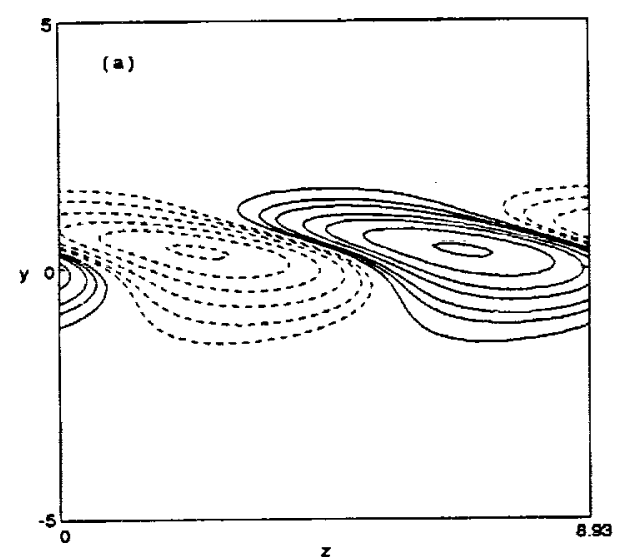

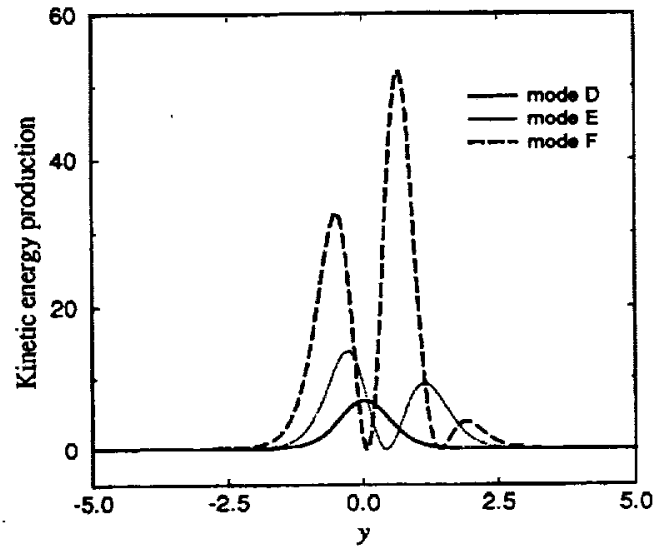

Figure 8. Profiles of the kinetic energy production for disturbance of zero-frequency and $\beta=0.7$. Case 2. $1 / R=0.05$

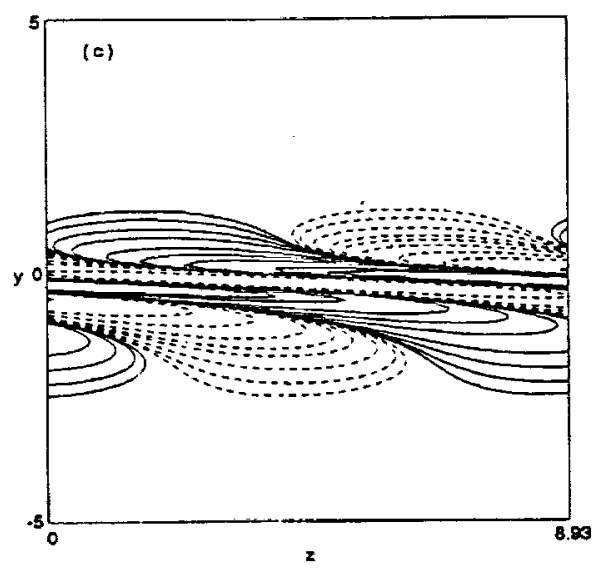

Figure 9. Comparison of the streamwise vorticity $\omega_{x}$ of modes of $\beta=0.7$ of zero and finite frequencies. Case 2. $1 / R=0.05$. (a) mode $\mathrm{A}$; (b) mode $\mathrm{B}$; (c) mode $\mathrm{C}$; (d) mode $\mathrm{D}$; (e) mode $\mathrm{E}$; (f) mode F; (g) mode G. 

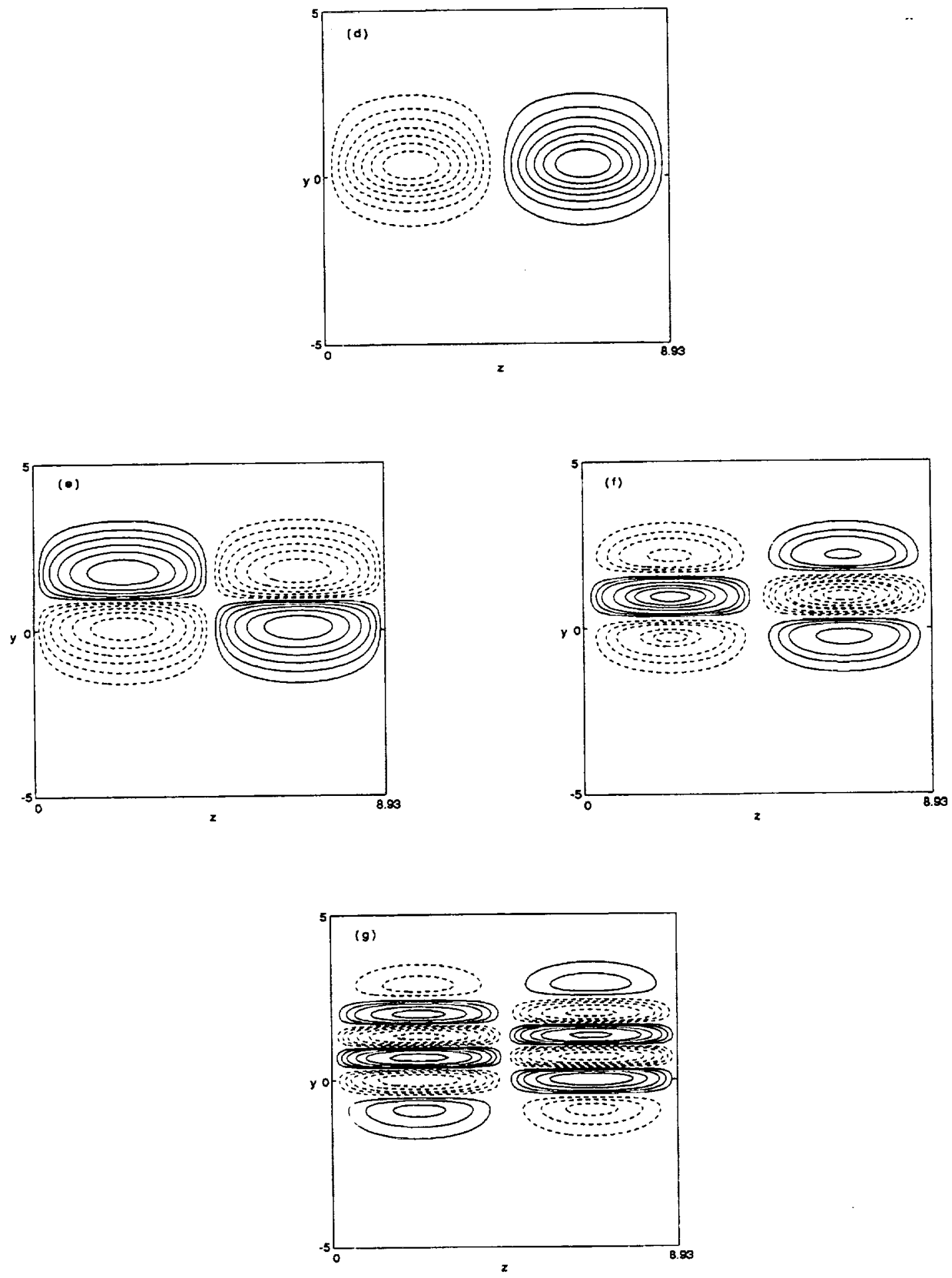

Figure 9. Continued. 

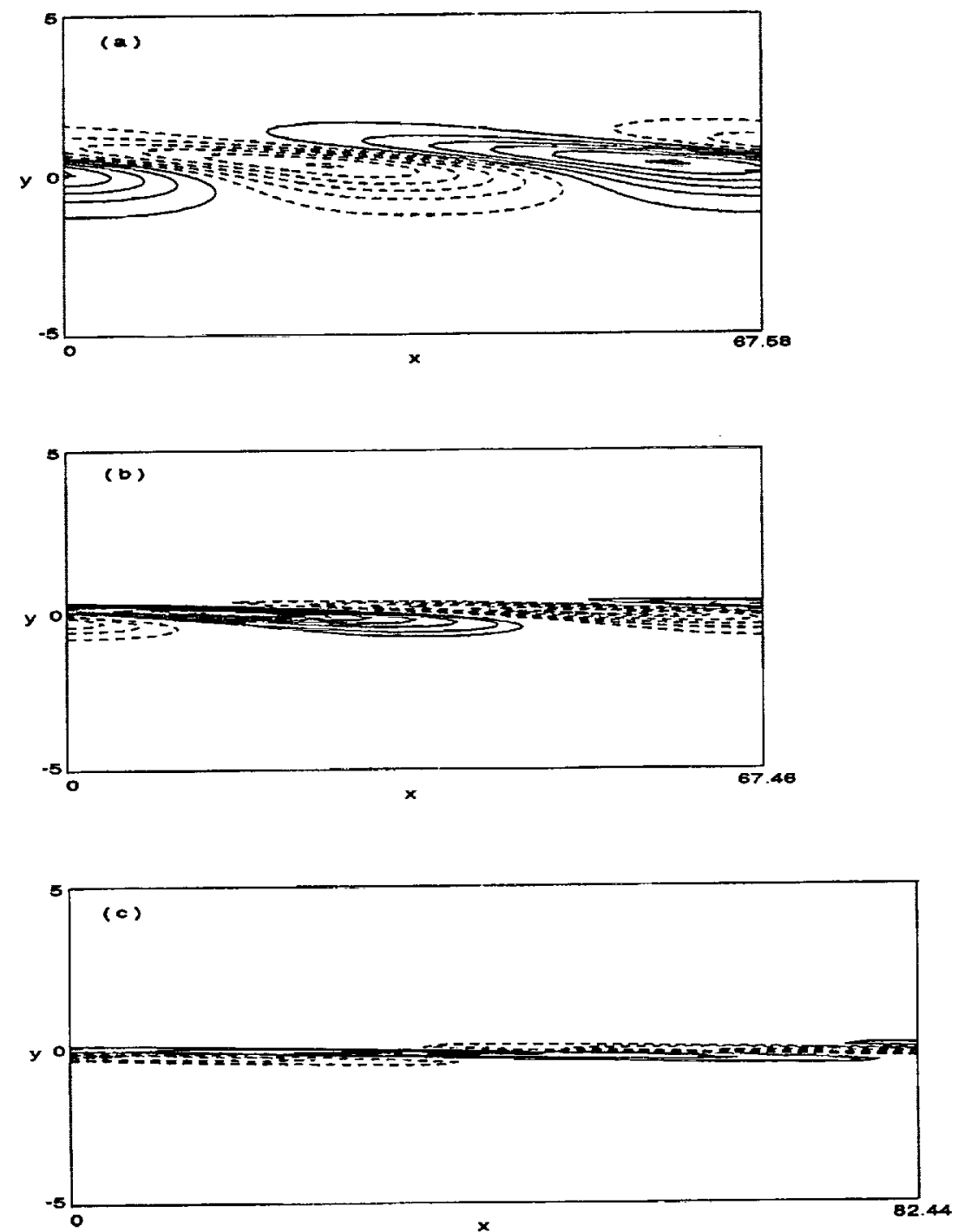

Figure 10. Comparison of the spanwise vorticity $\omega_{z}$ of finite frequency modes with $\beta=0.7$. Case 2 . $1 / R=0.05$. (a) mode A; (b) mode $B$; (c) mode $C$. 
Public reponting burden for this collection of information is estimated to average 1 houp per response, including the time lor reviewing instructions, searching existing data sources,

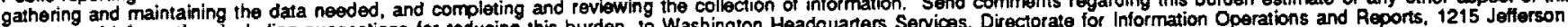

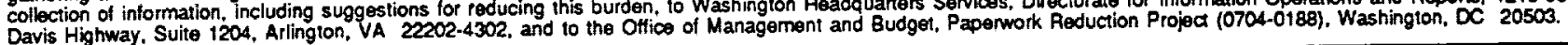

\begin{tabular}{|l|l|l|}
\hline 1. AGENCY USE ONLY (Leave blank) & $\begin{array}{c}\text { 2. REPORT DATE } \\
\text { July } 1993\end{array}$ & $\begin{array}{r}\text { 3. REPORT TYPE AND DATES COVERED } \\
\text { Technical Memorandum }\end{array}$
\end{tabular}

\section{TITLE AND SUBTITLE}

Linear Instability of Curved Free Shear Layers
5. FUNDING NUMBERS

WU-505-90-5K

\section{AUTHOR(S)}

William W. Liou

7. PERFORMING ORGANIZATION NAME(S) AND ADDRESS(ES)

National Aeronautics and Space Administration

Lewis Research Center

Cleveland, Ohio 44135-3191

9. SPONSORINGMONITORING AGENCY NAME(S) AND ADDRESS(ES)

National Aeronautics and Space Administration

Washington, D.C. 20546-0001
8. PERFORMING ORGANIZATION REPORT NUMBER

E-8027
10. SPONSORINGMONITORING AGENCY REPOAT NUMBER

NASA TM- 106290

AIAA-03252

ICOMP-93-28

CMOTT-93-11

11. SUPPLEMENTARY NOTES

Prepared for the 3rd Shear Flow Control Conference sponsored by the American Institute of Aeronautics and Astronautics, Orlando, Florida, July 6-9, 1993. William W. Liou, Institute for Computational Mechanics in Propulsion and Center for Modeling of Turbulence and Transition, NASA Lewis Research, (work funded under NASA Coperative Agreement NCC-3-233). ICOMP Program Director, Louis A. Povinelli, (216) 433-5818.

12a. DISTRIBUTIONAVAILABILTY STATEMENT

12b. DISTRIBUTION CODE

Unclassified - Unlimited

Subject Category 34

\section{ABSTRACT (Maximum 200 words)}

The linear inviscid hydrodynamic stability of slightly curved free mixing layers is studied in this paper. The disturbance equation is solved numerically using a shooting technique. Two mean velocity profiles that represent stably and unstably curved free mixing layers are considered. Results are shown for cases of five curvature Richardson numbers. The stability characteristics of the shear layer are found to vary significantly with the introduction of the curvature effects. The results also indicate that, in a manner similar to the Görtler vortices observed in a boundary layer along a concave wall, instability modes of spatially developing streamwise vortex pairs may appear in centrifugally unstable curved mixing layers.

\begin{tabular}{|c|c|}
\hline & $\begin{array}{c}\text { 15. NUMBER OF PAGES } \\
18\end{array}$ \\
\hline & $\begin{array}{r}\text { 16. PRICE CODE } \\
\mathrm{AO} 3\end{array}$ \\
\hline $\begin{array}{l}\text { 19. SECURITY CLASSIFICATION } \\
\text { OF ABSTRACT } \\
\text { Unclassified }\end{array}$ & 20. LIMTIATION OF ABSTRACT \\
\hline
\end{tabular}

